\title{
Formação dos Nomes Primitivos em Ikpeng
}

Formation of Primitive Noums in Ikpeng

\author{
Angela Fabiola Alves Chagas \\ Programa de Pós-Graduação em Letras (PPGL) \\ Universidade Federal do Pará (UFPA) \\ Kelly Edinéia Oliveria da Silva \\ Programa de Pós-Graduação em Letras (PPGL) \\ Universidade Federal do Pará (UFPA)
}

Resumo. O objetivo deste artigo é descrever como os nomes primitivos são gerados na língua lkpeng (Karib). Esta língua possui dois tipos de nomes: os possuíveis e os não-possuíveis, existindo distinções morfológicas e semânticas entre ambos. Morfologicamente, os possuíveis podem carregar prefixos de pessoa, que indicam a figura do possuidor e um sufixo relacional que indica a função genitiva; enquanto os últimos não podem ter nenhum desses morfemas afixados às suas bases. Os nomes possuíveis podem ser subdivididos em alienáveis e inalienáveis. Os primeiros, semanticamente, referem-se a objetos pessoais, enquanto os inalienáveis, geralmente se relacionam semanticamente a noções como: partes do corpo, termos de parentesco e sentimentos ou emoções e, ocasionalmente, podem ocorrer sem indicação do possuidor, tal como os alienáveis. Na forma absoluta, além da morfologia que indica essa condição, ambos podem se manifestar através de uma raiz supletiva. Os nomes não-possuíveis semanticamente relacionam-se a elementos da natureza e não carregam morfologia genitiva.

Palavras-chave: Nomes; Morfologia Nominal; Língua Ikpeng.

\begin{abstract}
The aim of this article is to describe how primitive noums are formed in the lkpeng language (Karib). This language has two types of noums: the possessed and the non-possessed, with morphological and semantic distinctions between them. Morphologically, the possessed ones can carry personal prefixes, which indicate the figure of the possessor, and a relational suffix that indicates the genitive function; while the latter cannot have any of these morphemes affixed to their roots. The possessed noums can be subdivided into alienables and inalienables. The formers, semantically, refer to personal objects, while the inalienable ones, usually, are semantically related to notions such as: body parts, kinship terms and feelings or emotions and, occasionally, they can occur without indication of the possessor, such as alienable noums. In an absolute form, both can be realized by supletive roots. Non-possessed noums semantically relate to elements of nature and do not carry any genitive morphology.
\end{abstract}

Keywords: Noums; Nominal Morphology; Ikpeng Language

\section{INTRODUÇÃO}

O objetivo deste artigo é descrever como os nomes primitivos são formados na língua Ikpeng, pertencente ao Ramo Pekodiano da família linguística Karib, ao lado do Bakairi e do Arara do Pará, seu provável co-dialeto (MEIRA; FRNACHETTO, 2005). O Ikpeng é falado no estado do Mato Grosso, no Parque Indígena do Xingu, por uma população de aproximadamente 500 indivíduos que residem atualmente nas aldeias Moygu, Rawo, Arayo e Tupara. 
Os dados desta pesquisa foram coletados por Chagas em viagens de campo realizadas entre os anos de 2009 a 2020 na aldeia Moygu - em que habita mais de cinquenta por cento da população lkpeng -e são provenientes tanto de elicitação, quanto de textos orais.

De acordo com os dados analisados, pode-se dizer que existem dois tipos de nomes em Ikpeng: os possuíveis e os não-possuíveis, tal como já observado por Pachêco, (2001, p. 93). As principais distinções entre ambos consistem em que, morfologicamente, os primeiros podem carregar prefixos de pessoa, que indicam a figura do possuidor e um sufixo relacional que indica a função genitiva; enquanto os últimos não podem ter nenhum desses morfemas afixados às suas bases. Os nomes possuiveis podem ser subdivididos em alienáveis e inalienáveis. Os primeiros, semanticamente, referem-se a objetos pessoais; já os nomes inalienáveis, geralmente se relacionam, semanticamente, a noções como: partes do corpo, termos de parentesco e sentimentos ou emoções. Ocasionalmente, os nomes possuíveis podem ocorrer numa forma absoluta, em que não há indicação do possuidor, com adição de morfologia específica para indicar essa condição; além disso, podem também apresentar raízes supletivas. Os nomes não-possuíveis semanticamente relacionam-se a elementos da natureza e não carregam morfologia genitiva.

\section{MORFOLOGIA DOS NOMES POSSUÍVEIS}

Os nomes possuíveis opõem-se quanto à alienabilidade (alienáveis vs inalienáveis), existindo distinções semânticas e gramaticais entre eles - no último caso, quando se apresentam na forma absoluta. Na forma flexionada, ambos possuem a mesma estrutura, qual seja: um prefixo de pessoa que indica a figura do possuidor e um sufixo relacional que indica o genitivo agregados à raiz, podendo opcionalmente carregar o morfema de número plural \{-kom $\sim-n g m o\}{ }^{1}$, quando necessário: [PESS- $\sqrt{ } \ldots$ GEN(-PL)].

Os prefixos de pessoa que indicam o possuidor nos nomes são os da série II, que nos verbos transitivos indicam a marcação do objeto²:

Tabela 01: Prefixos Pessoais da língua Ikpeng

\begin{tabular}{|c|c|c|c|c|}
\hline \multirow{3}{*}{ PESSOA } & \multicolumn{4}{|c|}{ VERBO TRANSITIVO } \\
\hline & \multicolumn{2}{|c|}{$\begin{array}{c}\text { Série I } \\
\text { (Função A) }\end{array}$} & \multicolumn{2}{|c|}{$\begin{array}{c}\text { Série II } \\
\text { (Função P) }\end{array}$} \\
\hline & C- Inicial & V-Inicial & C- Inicial & V-Inicial \\
\hline 1 & \multicolumn{2}{|l|}{ ye- } & \multicolumn{2}{|c|}{$g-$} \\
\hline 2 & \multicolumn{2}{|c|}{$m-$} & \multicolumn{2}{|c|}{$w-$} \\
\hline $1+2$ & \multicolumn{2}{|c|}{ kut- } & $u k-/ w i ̈-$ & $u g(w)-$ \\
\hline $\begin{array}{l}3 \\
3 \operatorname{Ref}\end{array}$ & \multicolumn{2}{|c|}{$\varnothing-$} & $\begin{array}{l}i- \\
t i-\end{array}$ & $\begin{array}{l}y- \\
t-\end{array}$ \\
\hline
\end{tabular}

Fonte: adaptado de Chagas (2013, p. 174)

1 A alomorfia do morfema de número em Ikpeng é condicionada pela natureza (vocálica ou consonantal) do último segmento da base onde o morfema irá se agregar. Em radicais C-finais, realiza-se como \{-kom\} e em $V$-finais, assume a forma \{-ngmo\}. Esse morfema pode flexionar nomes e verbos nesta língua.

2 A mesma série de prefixos pessoais também pode ocorrer nas posposições. 
Apresentamos abaixo dois paradigmas nominais $\sqrt{ } p u$ 'pé' (C-inicial) e $\sqrt{ } a m o$ 'ombro' (V-inicial) para ilustrar a ocorrência dos prefixos de pessoa da série II:

$\begin{array}{llcl}\text { (1) } & \text { pu } \quad \text { 'pé' } & \text { (2) } & \text { amo 'ombro' } \\ 1 & i-p u-n & & \text {-amo-rï } \\ 2 & o-p u-n & & \text { w-amo-rï } \\ 1+2 & w i-p u-n & & \text { ugw-amo-rï } \\ 3 & i-p u-n & & \text {-amo-rï } \\ 3 \text { Ref } & t i-p u-n & & \text { tamo-rï }\end{array}$

O morfema relacional que indica o genitivo se realiza através dos seguintes alomorfes: $\{-t\},\{-n\},\{-r i\}$, $\{-r u\},\{-t x i\}$ e $\{-\varnothing\}$, cuja distribuição nas raízes não é previsível nem semântica nem fonologicamente. Abaixo, damos alguns exemplos de uso desses morfemas.

\begin{tabular}{|c|c|c|}
\hline & $\begin{array}{l}g \text {-eretpu-t } \\
\text { 1-cabelo-GEN }\end{array}$ & 'meu cabelo' \\
\hline & $\begin{array}{l}\text { ï-moropo-n } \\
1 \text {-bolsa -GEN }\end{array}$ & 'minha bolsa' \\
\hline & $\begin{array}{l}\ddot{I} \text {-manga-rï } \\
\text { 1-seio-GEN }\end{array}$ & 'meu seio' \\
\hline & $\begin{array}{l}\text { g-eng-ru3 } \\
\text { 1-olho-GEN }\end{array}$ & 'meu olho' \\
\hline & $\begin{array}{l}\text { ï-rop-txi'meu a } \\
\text { 1-arco-GEN }\end{array}$ & \\
\hline & $\begin{array}{l}\text { g-aginu- } \varnothing \\
\text { 1-choro-GEN }\end{array}$ & 'meu choro' \\
\hline
\end{tabular}

Os nomes possuíveis podem também ocorrersem ser numa forma possuída. Nesses casos, não carregam os morfemas de pessoa nem os morfemas genitivos mencionados acima. Nessas circunstâncias, eles são marcados com as formas\{-ngo - Ø\}, como pode ser visto abaixo:

(9) $\begin{aligned} & \text { eretpu-ngo } \\ & \text { cabelo-ABS }\end{aligned}$
(10) $\begin{aligned} & \text { moropo- } \varnothing \quad \text { 'bolselo' } \\ & \text { bolsa-ABS }\end{aligned}$

3 Conforme já mencionado, não é possível prever a realização dos morfemas indicadores de genitivo. No entanto, é preciso dizer que o alomorfe $\{-r u\}$ é frequentemente encontrado em raízes terminadas em consoante velar (ex: mingru 'sangue', txigru 'urina', ewrogru 'flor', myangru 'preguiça', epragru 'estomago'). Em alguns casos, a forma em questão parece ter sido reinterpretada pelas falantes nativos como parte da raiz, não sendo mais possível a sua segmentação. Ambas as situações precisam ser melhor investigadas na língua. 
(11) manga- $\varnothing \quad$ 'seio'

seio-ABS

(12) ongru-ngo 'olho'

1-olho-ABS

(13) topkak- $\varnothing \quad$ 'arco'

$\operatorname{arco-ABS}$

(14) aginu-ngo 'choro, lágrima'

choro-ABS

Tal como já dito, há distribuição semântica entre os dois sub-tipos de nomes possuíveis, já que os nomes alienáveis se referem a objetos pessoais e os inalienáveis a partes do corpo, termos de parentesco e sentimentos ou emoções. Morfologicamente, comportam-se da mesma forma, podendo apresentar-se

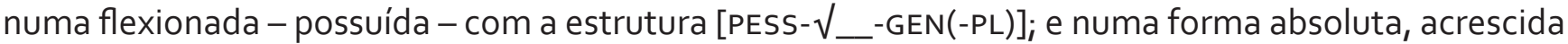
do morfema que indica que o nome não está sendo possuído na ocasião: $\left[\sqrt{ } \_\right.$_-ABS(-PL)]. Destacamos que alguns nomes possuíveis apresentam raízes supletivas, quando não possuídos. Apresentamos, abaixo, alguns exemplos de nomes possuíveis com raízes supletivas ocorrendo em sua forma possuída (exemplos a.) e constrastivamente na forma absoluta (exemplos b.).

a. g-en- $\varnothing \quad$ 'meu dente'

a. ï-pu-n 'meu pé'

a. $g$-ere- $t$

a. g-emi-n

a. g-erempiu- $n$

a. $\ddot{\imath}-m u-\varnothing$

a. g-ayo-n

a. g-ew-ri

a. g-apo-n 'meu nome'

'minha fome'

'minha garganta'

'meu ovo'

'minha cuia'

'minha casa'

'minha borduna' b. were-ngo 'dente'

b. pugu-ngo 'pé'

b. ore-ngo 'nome'

b. omi-ngo 'fome'

b. gegï- $\varnothing \quad$ 'garganta'

b. mumu- $\varnothing$ 'ovo'

b. wayo- $\varnothing$ 'cuia'

b. owro- $\varnothing$ 'casa'

b. opo- $\varnothing \quad$ 'borduna'

Quanto ao seu processo de formação, grande parte dos nomes possuíveis é formado por uma raiz sem categoria gramatical mais um morfema categorizador ( $\sqrt{ }_{\text {_-NMLZ}}$ ), que dá origem a uma base nominal que, por sua vez, pode carregar os morfemas flexionais (pessoa e número) ou dar origem a outras palavras através de processos de derivação. Essas bases nominais podem gerar outros nomes, bem como verbos e adjetivos na língua Ikpeng. No entanto, a formação de nomes derivados, verbos e adjetivos a partir de nomes está fora do escopo deste trabalho.

Dadas as informações acima, apresentamos, a seguir, nossa proposta de formação dos nomes possuíveis, na qual, consideramos os morfemas genitivos como os responsáveis pela atribuição de categoria nominal às raízes às quais se agregam, uma vez que sua realização é obrigatória e exclusiva nos nomes 4 . Consideramos que a raiz concatenada ao morfema genitivo dá origem a um radical nominal que pode receber a morfologia flexional de pessoa e número. Utilizaremos as palavras abaixo para ilustrar a formação dos nomes possuíveis (alienáveis e inalienáveis) em Ikpeng.

4 Estamos incluindo aqui sob o rótulo de morfema genitivo também as formas $\{-n g o \sim-\varnothing\}$ que indicam a ocorrência dos nomes possuíveis na forma absoluta, isto é, sem a especificação do possuidor. 
Forma Flexionada

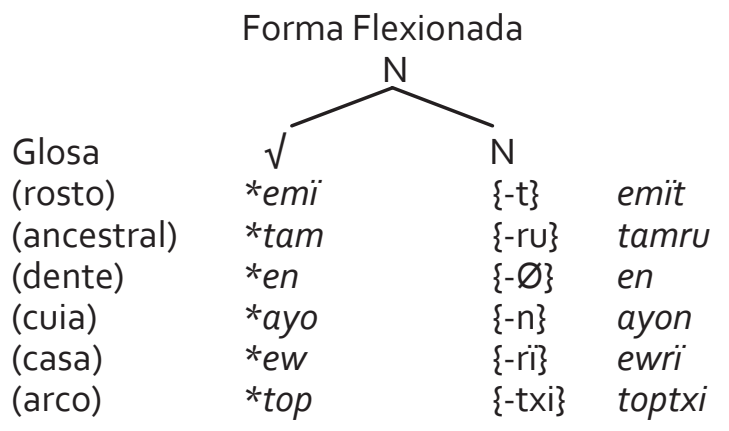

Forma Absoluta

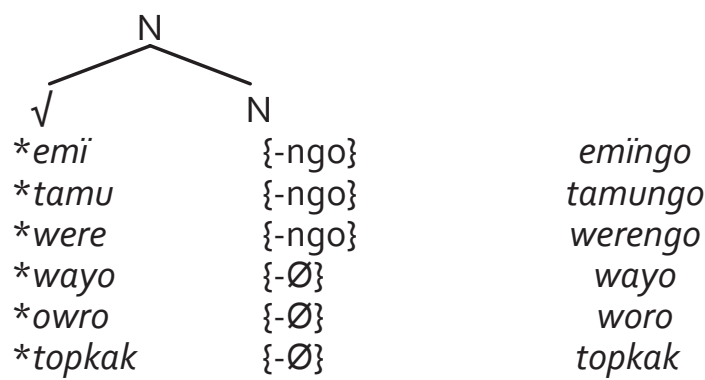

É importante mencionar que as formas absolutas, tal como se encontram já são interpretadas como palavras plenas pelos falantes nativos da língua Ikpeng, dado que não necessitam de nenhum outro morfema para sua utilização; ao contrário da forma flexionada, que precisa (i) ou carregar um prefixo de pessoa que indica a figura do possuidor, (ii) ou indicar o possuir sintaticamente através de um nome ou pronome, caso em que é dispensada a ocorrência do prefixo ${ }^{5}$. Apresentaremos abaixo exemplos de uso dessas palavras na forma flexionada (exemplos a.) e, em seguida, na sua forma absoluta (exemplos b).

a. Karake nen gemït

karake nen g-emï-t

bom/bonito DEM 1P-rosto-GEN

'Meu rosto é bonito'

b. Emïngo karake enengkili

emï-ngo karake $\varnothing$-enengki-lï

rosto-ABS bom/bonito 3A-aparecer-PAS.IM

'O rosto bonito apareceu'

(25)

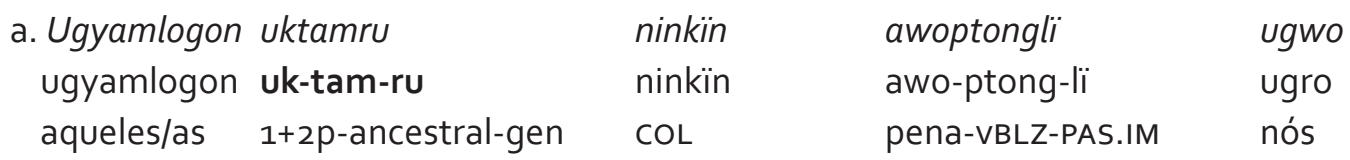

'Foram aqueles nossos ancestrais que nos criaram das penas/flechas'

[Pïrom Miran - Origem da Flecha]
b. Egemelan
tamungongmo
e-ke-me-lan
tamu-ngo-ngmo
3A-fazer-CAUS-PAS.IM ancestral-ABS-PL
'Os ancestrais fizeram'

$\begin{array}{lllll}\text { a. Ugwen } & \text { ke } & \text { elip } & \text { man imo } & \text { Wayman } \\ \text { ugw-en- } \varnothing & \text { ke } & \text { elïp man imro } & \text { wayman } \\ \text { 1+2P-dente-GEN } & \text { POSP:com } & \text { colar } & \text { PART ele } & \text { jaboti } \\ \text { 'Ele, o Wayman (jaboti), fazia colar com os nossos dentes' } & \end{array}$

[Wayman Miran - História do Jaboti] 5 Como o objetivo deste artigo é apresentar o processo de formação dos nomes, não ampliaremos a discussão nem apresentaremos exemplos da realização sintática da figura do possuidor. 


$\begin{array}{clll}\text { b. Orong } & \text { pok } & \text { tong } & \text { werengo } \\ \text { orong } & \text { pok } & \text { tong } & \text { were-ngo } \\ \text { chão } & \text { POSP:em } & \text { IDEO:Som.de.cair } & \text { dente-ABS }\end{array}$

'O dente caiu no chão'

(27)

a. Manumtxi gayon

m-anum-txi g-ayo-n

2A-carregar-N.PAS IP-cuia-GEN

'Você carregará minha cuia'

b. Yapkoteli wayo

y-apkote-lï wayo- $\varnothing$

1A-quebrar-PAS.IM cuia-ABS

'Eu quebrei a cuia'

(28)

$\begin{array}{lll}\text { a. Karanang } & \text { gewrï } & \text { paraktxi } \\ \text { k-ar-an-nang } & \text { g-ew-rï } & \text { paraktxi } \\ \text { 1P-INTR-ir-PROG } & \text { 1P-casa-GEN } & \text { POSP:para.dentro.de } \\ \text { 'Eu estou indo para minha casa' } & \end{array}$

b. Owro warap tigït man imo man totu

owro- $\varnothing$ parap tïgït man imro man totu

casa-ABS POSP:dentro.de escuropART ele PART IDEO:som.de.caminhar

'Quando ele caminhou para dentro da casa, já estava escuro'

[Okoloy Mïran - História do Mel]

(29)

\begin{tabular}{|c|c|c|c|c|}
\hline a. Ime & ïprï & ïprï & ïroptxi & ïroptxi \\
\hline ime & ï-p-rï & ï-p-rï & ï-rop-txi & ï-rop-txi \\
\hline mãe(voc) & 1-flecha-GEN & 1-flecha-GEN & 1-arco-GEN & 1-arco-GEN \\
\hline
\end{tabular}

[Wayman Miran - História do Jaboti]

$\begin{array}{llll}\text { b. Topkak } & \text { nem } & \text { tximna } & \text { napli } \\ \text { topkak } & \text { nem } & \text { tximna } & \text { naplï } \\ \text { arco } & \text { DEM } & 1+3 & \text { instrumento }\end{array}$

'O arco é nosso instrumento'.

\section{Morfologia dos Nomes Não-Possuiveis}

Como já mencionado, os nomes não-possuiveis não carregam morfologia aparente. Portanto, nossa proposta de análise para eles é que esses nomes são formados também por raízes desprovidas de classe gramatical somada a um morfema categorizador fonologicamente nulo $\left(\sqrt{ } \_-\varnothing\right)$. A título de exemplo, 
mostramos abaixo a formação de três nomes não-possuídos ototo 'tucunzeiro', kulu 'formiga' e anma 'caminho', como representantes dos demais.

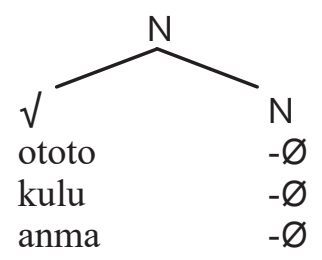

(30)

$$
\begin{array}{ll}
\text { Oterelan } & \text { ototo } \\
\text { ot-ere-lan } & \text { ototo } \\
\text { INTR-acender-PAS.IM } & \text { tucunzeiro } \\
\text { 'O tucunzeiro acendeu/pegou fogo' }
\end{array}
$$

[Okoloy Mirran - "História do Mel"]

(31) Kulu kurakta

kulu kur-ak-ta

formiga 1+2-comer-CONV

'Vamos comer formiga'

[Puron Miran - "História do Sapo"]

(32)

$\begin{array}{llllll}\text { Owrotpo } & \text { warako } & \text { anma } & \text { warako } & \text { ompan } & \text { irwako } \\ \text { owrotpo } & \text { warako } & \text { anma } & \text { warako } & \text { ompan } & \text { irwako } \\ \text { casa.velha } & \text { POSP:dentro } & \text { caminho } & \text { POSP:dentro } & \text { todo } & \text { mato }\end{array}$

'Agora você vai ficar numa casa velha, no caminho, em qualquer mato'

[Puron Miran - "História do Sapo"]

\section{Paradigmas Nominais}

Levando em consideração os aspectos gramaticais pessoa, número e posse, o paradigma dos nomes possuíveis em Ikpeng apresenta as treze formas abaixo. Observe que o radical ( $V_{-}$-GEN) da palavra montxi 'cabeça' inicia com consoante e termina com vogal, ao contrário de eret 'nome', que inicia por vogal e finda com consoante. Esses dados evidenciam a alomorfia fonologicamente condicionada pelos segmentos iniciais e finais dos temas nominais, tanto dos prefixos de pessoa, quanto do sufixo de número.

$\begin{array}{lllll}\text { 1 SG } & \text { ï-montxi- } \varnothing & \text { 'minha cabeça' } & \text {-ere-t } & \text { 'meu nome' } \\ \text { 2 SG } & 0 \text {-montxi- } \varnothing & \text { 'tua cabeça' } & \text { w-ere-t } & \text { 'teu nome' } \\ \text { 2 PL } & 0 \text {-montxi- } \varnothing \text {-ngmo } & \text { 'cabeças de vocês' } & \text { w-ere-t-kom } & \text { 'nomes de vocês' } \\ \text { 1+2 SG } & \text { wï-montxi- } \varnothing & \text { 'nossa cabeça' } & \text { ugw-ere-t } & \text { 'nosso nome' } \\ \text { 1+2 PL } & \text { wï-montxi- } \varnothing \text {-ngmo } & \text { 'nossas cabeças' } & \text { ugw-ere-t-kom } & \text { 'nossos nomes' } \\ \text { 3 SG } & \text { e-montxi- } \varnothing & \text { 'cabeça dele/a' } & \varnothing \text {-ere-t } & \text { 'nome dele/a' } \\ \text { 3 PL } & e \text {-montxi- } \varnothing \text {-ngmo } & \text { 'cabeças deles/as' } & \varnothing \text {-ere-t-kom } & \text { 'nomes deles/as' }\end{array}$




$\begin{array}{lllll}\text { 3REF SG } & \text { tï-montxi- } \varnothing & \text { 'sua própria cabeça' } & t \text {-ere-t } & \text { 'seu próprio nome' } \\ \text { 3REF PL } & \text { tï-montxi- } \varnothing \text {-ngmo } & \text { 'suas próprias cabeças' } & t \text {-ere-t-kom } & \text { 'seus próprios nomes' } \\ \text { NP SG } & \text { NP montxi- } \varnothing & \text { 'cabeça de NP' } & \text { NPere-t } & \text { 'nome de NP' } \\ \text { NP PL } & \text { NP montxi- } \varnothing \text {-ngmo } & \text { 'cabeças de NP' } & \text { NPere-t-kom } & \text { 'nomes de NP' } \\ \text { ABSOL SG } & \text { montxi-ngo } & \text { 'cabeça' } & \text { ore-ngo } & \text { 'nome' } \\ \text { ABSOL PL } & \text { montxi-ngo-ngmo } & \text { 'cabeças' } & \text { ore-ngo-ngmo } & \text { 'nomes' }\end{array}$

\section{Considerações Finais}

Neste artigo, fizemos uma breve descrição da formação de nomes primitivos na língua lkpeng. Mostramos que os nomes possuíveis e não-possuiveis são semelhantes quanto à sua estrutura interna. Ambos são formados a partir de raízes sem categoria gramatical, acrescida de um morfema categorizador [ $V_{\text {__}}$-NMLZ], que no caso dos nomes possuíveis, consideramos ser os sufixos relacionais genitivos $\{-t\},\{-n\},\{-r i\},\{-r u\},\{-t \times\}$ e $\{-\varnothing\}$. Os nomes possuíveis (alienáveis e inalienáveis) podem ser flexionados em pessoa - através dos prefixos pessoais da Série II - e número \{-kom -ngmo\}, sendo realizados através da estrutura [PESS- $\sqrt{ }$ __-GEN(-PL)], em que a raiz carrega as informações semânticas e fonológicas referentes ao objeto possuído, o prefixo de pessoa indica a figura do possuidor, o sufixo genitivo indica que o nome está sendo possuído naquela circunstância e o sufixo de número, quando presente, indica mais de um objeto sendo possuído. Eventualmente, esses nomes podem ocorrer de maneira não-possuída, realizando-se numa forma absoluta. Nesses casos, não podem carregar prefixo de pessoa e no lugar dos morfemas genitivos, apresentam uma morfologia específica $\{-n g o \sim \varnothing\}$ que, semanticamente, indica que o nome não pertence a ninguém em dada situação, apresentando a estrutura $\left[\sqrt{ } \_\right.$_-ABS $\left.(-\mathrm{PL})\right]$. Além disso, algumas raízes apresentam modificação fonológica em suas formas.

\section{Lista De Glosas}

\begin{tabular}{ll|ll}
1 & primeira pessoa & INTR & intransitivo \\
$1+2$ & primeira pessoa inclusiva & N.PAS & não-passado \\
$1+3$ & primeira pessoa exclusiva & NMLZ & nominalizador \\
2 & segunda pessoa & P & objeto \\
3 & terceira pessoa & PART & partícula \\
A & sujeito & PAS.IM & passado imediato \\
ABS & forma absoluta & PESS & pessoa \\
CAUS & causativo & PL & plural \\
COL & coletivo & POSP & posposição \\
CONV & convite & PROG & progressivo \\
DEM & pronome demonstrativo & REF & reflexivo \\
GEN & genitivo & VBLZ & verbalizador \\
IDEO & ideofone & VOC & vocativo
\end{tabular}




\section{Referências}

CHAGAS, A. O Verbo Ikpeng: estudo morfossintático e semântico-lexical. 2013. Tese (Doutorado em Linguística). Instituto de Estudos da Linguagem, Universidade Estadual de Campinas, Campinas, 2013. MEIRA, Sérgio; FRANCHETTO, Bruna. The Southern Carib Languages and the Cariban Family. International Journal of American Linguistics, vol 7, n. 2, 127-190. Chicago: Chicago University Press, 2005.

PACHÊCO, Frantomé. Morfossintaxe do verbo Ikpeng (Karib). Tese de Doutorado Campinas: UNICAMP, 2001. 